\title{
Enabling aberration retrieval of microlenses with the Extended Nijboer-Zernike (ENZ) diffraction theory
}

\author{
Sven van Haver, Joseph J.M. Braat, Silvania F. Pereira \\ Optics Research Group, Department of Imaging Science and Technology, \\ Faculty of Applied Sciences, Technical University Delft, \\ Lorentzweg 1, NL-2628 CJ Delft, The Netherlands
}

\begin{abstract}
We propose a measurement approach that allows the determination of aberrations of a microlens by analyzing the through-focus intensity image it produces when the object is a point source. To simulate image formation by a microlens we apply the extended version of the Nijboer-Zernike diffraction theory (ENZ) that uses the Debye diffraction integral to compute the image point-spread function. Due to the aperture size of the microlens and the finite dimensions of the pixels of the electronic detector the Debye diffraction integral should be adapted according to the Li-Wolf scaling rules to yield correct results. In addition to this we also discuss the experimental requirements posed by this characterization approach and derive from this a suitable experimental setup.
\end{abstract}

Keywords: Diffraction, point-spread function, Rayleigh integral, Debye integral, microlens, characterization, aberration retrieval

\section{INTRODUCTION}

In the literature and in practice, many different approaches exist to determine the quality of imaging systems. Wavefront analysis of the optical field in the exit pupil of the imaging system is the most common method. With interferometry or with wavefront slope detection (Shack-Hartmann method), precise data about the wavefront aberration introduced by the lens can be obtained. Using this data, an accurate prediction of the image quality of the system is possible. To this goal, the light propagation from the exit pupil to the image region is calculated with the aid of an adequate optical diffraction model. Other methods for quality assessment use spatial frequency analysis by measuring the contrast and phase of the optical transfer function of the lens. From the measured transfer, it is basically possible to go back to the wavefront in the exit pupil of the system and to know the aberrational state of the system. The object structures to be imaged are periodic; they can also be composed of some finite band of spatial frequencies.

In this paper we focus on a different approach, namely the analysis of the through-focus intensity distribution in the image region of the optical system. The object is preferably a point source and we measure the threedimensional point-spread function on the image side. Although the measurement of the intensity does not allow the retrieval of the phase of the focused field, methods have been developed to retrieve the phase function in the exit pupil of the optical system from intensity measurements in the image region. ${ }^{1}$ In practice, one proceeds in an iterative way to find the amplitude and phase in the exit pupil of the imaging system. With a first guess for the exit pupil function, one calculates the image intensity in a particular plane. From the differences with respect to the measured intensity, an updated pupil function is constructed. This procedure is repeated until convergence is detected for the complex exit pupil function. An improvement of this method is obtained by considering the image intensity distribution in several defocused image planes. The forward and backward wave propagation from exit pupil to image plane is, for sufficiently low numerical aperture, based on a scalar diffraction integral. For systems with high numerical aperture, NA $>0.6$, a vector diffraction model is required. The method we have adopted for the wave propagation from entrance pupil to image region is based on the extended NijboerZernike aberration theory (ENZ). Using this diffraction theory, we have at our disposal a semi-analytic solution of the Debye diffraction integral for an imaging system with a pupil function characterized by phase deviations

\footnotetext{
Further author information: (Send correspondence to Sven van Haver)

Sven van Haver: E-mail: s.vanhaver@tudelft.nl, Telephone: +31 152782116
}

Optical Modelling and Design, edited by Frank Wyrowski, John T. Sheridan, Jani Tervo, Youri Meuret, Proc. of SPIE Vol. 7717,77170 - ( ) 2010 SPIE · CCC code: 0277-786X/10/\$18 · doi: 10.1117/12.854412 
$\Phi$ (aberration) and by possible amplitude defects. Such a transmission function of the optical system is mapped into a single complex function

$$
g(\rho, \theta)=A(\rho, \theta) \exp \{i \Phi(\rho, \theta)\},
$$

with $\rho$ and $\theta$ the normalized radial and azimuthal coordinate on the exit pupil sphere, $\Phi(\rho, \theta)$ the phase deviation and $A(\rho, \theta)$ the possible transmission defects. This complex pupil function is then developed into a Zernike expansion with complex coefficients $\beta_{n}^{m}$ according to ${ }^{1}$

$$
A(\rho, \theta) \exp \{i \Phi(\rho, \theta)\}=\sum_{n, m} \beta_{n}^{m} R_{n}^{|m|}(\rho) \exp (i m \theta) .
$$

With $R_{n}^{m}(\rho)$ the radial part of the Zernike polynomial of degree $n$ and azimuthal order $|m|$, for $n \geq 0$ and $n-|m| \geq 0$ and even. Note that the azimuthal parameter $m$ can take both positive and negative integer values.

The basic principles of the extended Nijboer-Zernike diffraction theory have been given in two papers published in $2002 .^{2,3}$ The ENZ reconstruction method of the pupil function, using a series of through-focus intensity patterns, has been described in several publications. ${ }^{1,4-6}$ The ENZ diffraction theory provides a solution of the Debye diffraction integral in terms of a series expansion with respect to the defocus parameter $f$ and the radial image coordinate $r$. The azimuthal dependence of a general defocused intensity pattern is given by means of a harmonic expansion with respect to the azimuthal image coordinate $\phi$. The Debye formulation of the diffraction integral is sufficiently accurate if the distance from exit pupil to image region tends to infinity (telecentric imaging condition). An other way of saying this is that the number of Fresnel zones in the exit pupil should be sufficiently large. ${ }^{7}$ The condition of a large Fresnel number is also easily satisfied if the imaging aperture is large, even when the exit pupil is at a finite distance.

The Debye integral does not produce reliable results for the image intensity if the numerical aperture becomes small or the distance from exit pupil to image plane is relatively small. In fact one should use in this case the Rayleigh formulation of the lens diffraction problem. This is illustrated in Figure 1 where we have plotted the through focus behavior of both the Rayleigh and Debye integrals for some relevant settings. In the case of microlenses we are in the regime displayed by Figures $1 \mathrm{~b}$ and $1 \mathrm{~d}$. These figures clearly illustrate that for optical configurations typical to micro-lenses the Debye integral is not a good representation of the more exact Rayleigh integral.

Fortunately, for a sufficiently small numerical aperture at the imaging side, the Rayleigh integral can be approximated by a scaled version of the Debye integral. ${ }^{8-10}$ Let us consider the optical configuration as depicted in Figure 2a. A plane wave is incident on a microlens array. The radiation from one array-element is focused in an intermediate focus and re-imaged by an auxiliary imaging lens. This re-imaging is necessary to allow for a magnification of the focal spot, that would otherwise only comprise a few pixels on the detector. Several defocused images are sequentially captured by a precise scanning in the axial direction $z$. In Figure $2 \mathrm{~b}$ the relevant distances are defined that determine whether the Debye approximation is valid or not. $z_{i}$ is the distance $E O^{\prime}$ from the exit pupil to the nominal image plane and $z$ the distance from the exit pupil to an actual defocused image plane. Without entering into detail, we give the scalar Debye diffraction integral for sufficiently small NA,

$$
\begin{array}{r}
A_{D}(r, \phi, f)=A_{0, D} \iint_{\text {pupil }} \exp \left\{-i f_{D} \rho^{2}\right\} E(\rho, \theta) \exp \{i \Phi(\rho, \theta)\} \times \\
\exp \left\{-i 2 \pi r_{D} \rho \cos (\theta-\phi)\right\} \rho d \rho d \theta,
\end{array}
$$

with the defocus parameter $f_{D}$ given by $f_{D}=\pi\left(z-z_{i}\right) s_{0}^{2} / \lambda$ and where the radial coordinate $r_{D}$ has been normalized by the diffraction unit $\lambda / s_{0}\left(s_{0}=\sin \alpha\right)$. The Debye integral approximates the Rayleigh integral at small values of the numerical aperture by replacing $A_{0, D}, f_{D}$ and $r_{D}$ by the following scaled quantities

$$
A_{0, R}=\frac{z_{i}}{z} A_{0, D} ; \quad f_{R}=\frac{z_{i}}{z} f_{D} ; \quad r_{R}=\frac{z_{i}}{z} r_{D},
$$

Applying these scaled quantities to generate adapted Debye curves for the different axes in Figure 1 results in curves that practically coincide with their Rayleigh counterparts and are therefore not included in the figures. 

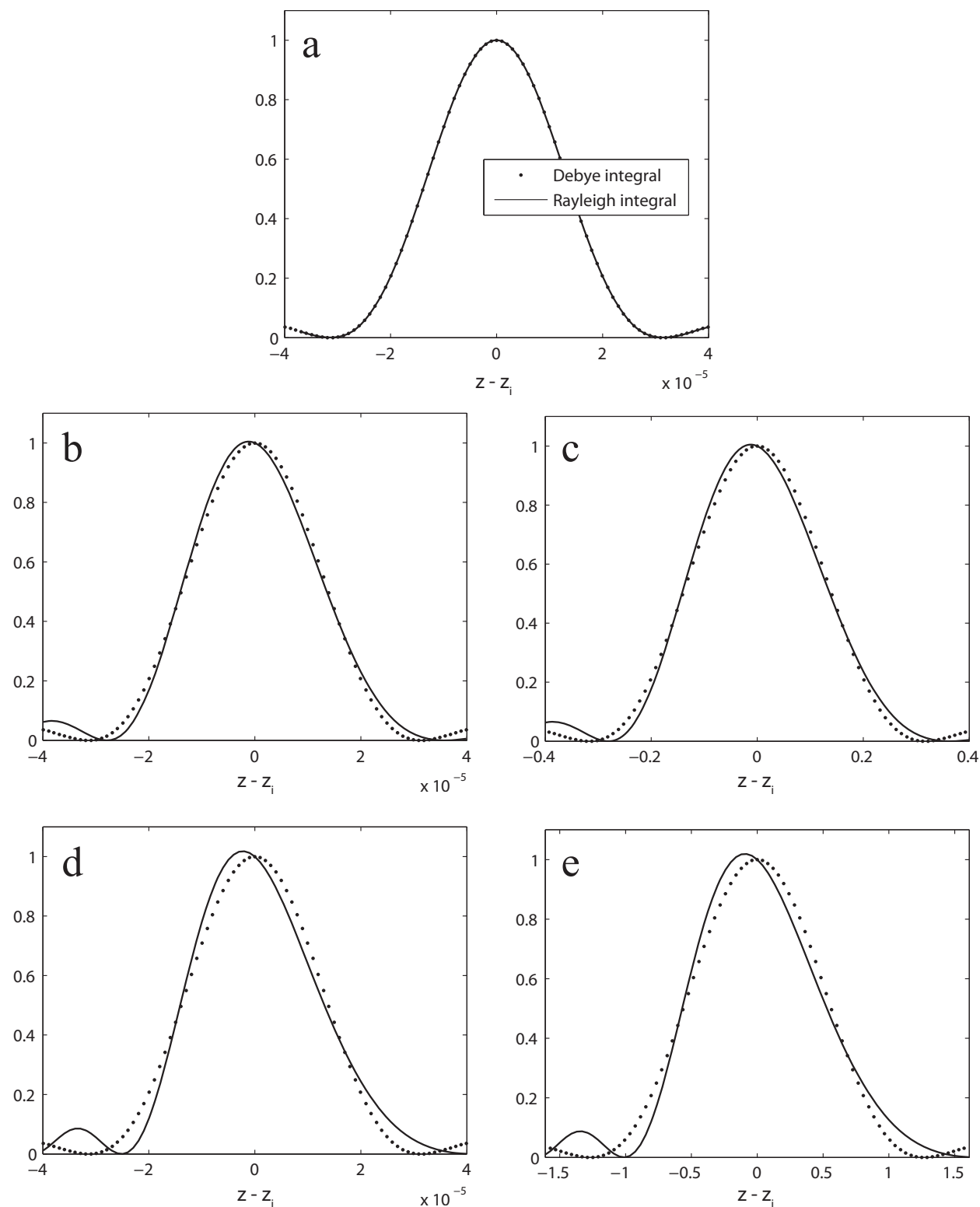

Figure 1. Comparison between the axial PSF intensity calculated according to both the Rayleigh and Debye integrals. Graph $a$ shows the PSF generated by a perfect, $1 \mathrm{~cm}$ diameter, lens ( NA $=0.2, \lambda=633 \mathrm{~nm}$ ) that is well within the Debye regime. In the four graphs below, we have started from the same settings as in $a$, however changing one parameter at the time (diameter of the lens or the NA) so that the resulting system is outside the Debye regime. In graphs b and d, we have changed the diameter to $100 \mu \mathrm{m}$ and $50 \mu \mathrm{m}$, respectively, and for graphs c and e, we have considered the NA value of 0.02 and 0.01 , respectively.

\section{MEASUREMENT APPROACH}

As already stated above our goal is to be able to reconstruct the aberration function of microlenses according to the approach developed in., ${ }^{1-6}$ This so-called ENZ aberration retrieval approach reconstructs the aberration function by analyzing the through-focus intensity distribution produced by the lens and matching this distribution by an analytic expression following from Debye diffraction theory. To successfully apply this same approach to microlenses we simply have to rescale the input variables of the standard Debye integral according to Eq.(4). 


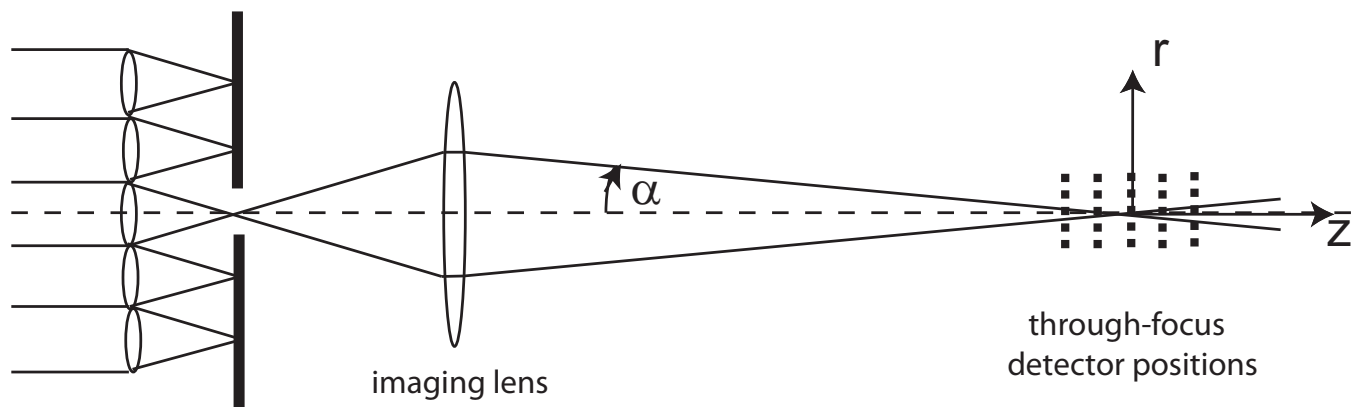

microlens array

a)

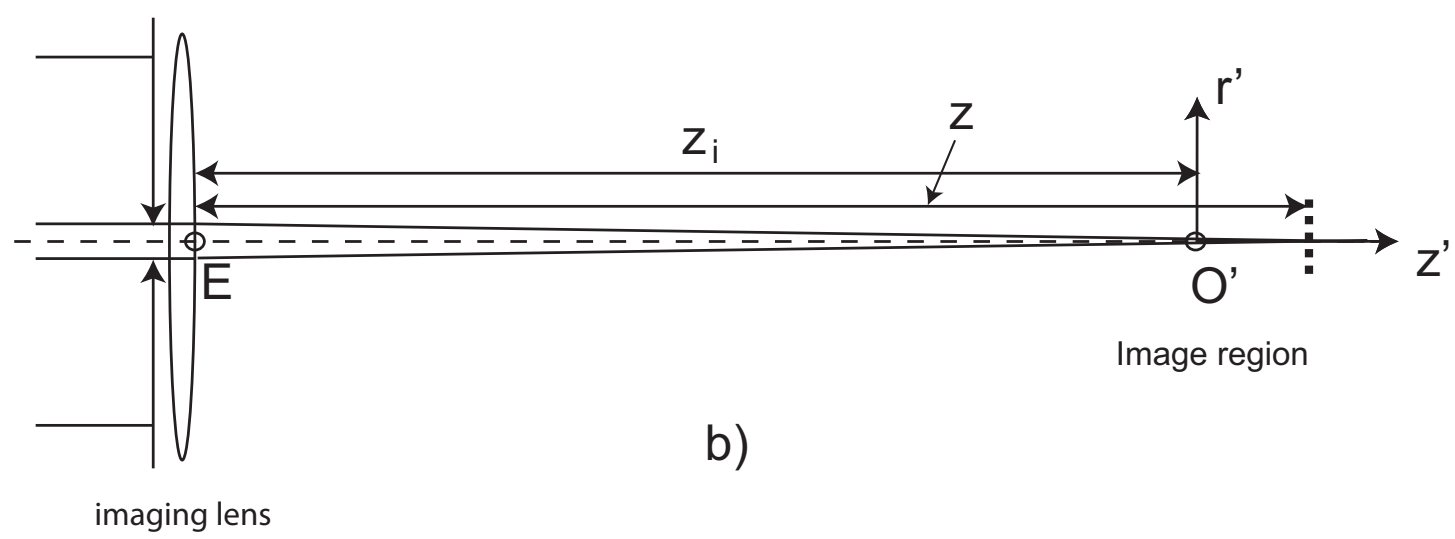

Figure 2. a) Schematic representation of the proposed microlens characterization approach. b) Definition of the relevant quantities that determine the applicability of the Debye approximation.

In this way no other adjustments to the ENZ aberration retrieval procedure are required and its full power can be equally well used in the characterization of microlenses. One thing should be kept in mind though, for the correction given in Eq.(4) we require accurate knowledge of the distances $z$ and $z_{i}$. These distances are sometimes hard to determine accurately as, for example, for an objective lens it is not always clear where the exit pupil is exactly located. Also, in the case that the PSF of the microlens is re-imaged with a certain magnification using several other lenses, exact knowledge on the paraxial configuration of this subsystem is required to achieve an accurate characterization of a microlens. Possible issues that can arise from this are discussed below when we describe our proposed set-up for microlens characterization.

\section{PROPOSED EXPERIMENTAL SETUP}

In order to design the experimental setup, we consider a given microlenses array. The array is from the Free University of Brussels; each microlens has a diameter of $242 \mu \mathrm{m}$ and a focal length of $0.8 \mathrm{~mm}$. In the picture shown in Fig. 3a we show a magnified image of the microlenses at their surface. Given the numerical aperture of the lens, one can predict that the point spread function will be of the order of $5 \mu \mathrm{m}(\lambda=633 \mathrm{~nm})$. This means that a direct measurement of the intensity of the electric field through focus is not possible using a CCD camera, given that its lateral resolution is of the order of a few microns. For this reason, an objective has to be used to magnify the focal region so that the lateral spread of the image of the focused beam would fill an area of about 100x100 pixels. With this configuration, one should keep in mind that in order to isolate the effect of the aberrations of the microlenses and that of the objective in the through focus images, the aberrations of the objective should be well known. Also, the numerical aperture of the objective should be larger than that of the microlenses. 
a)

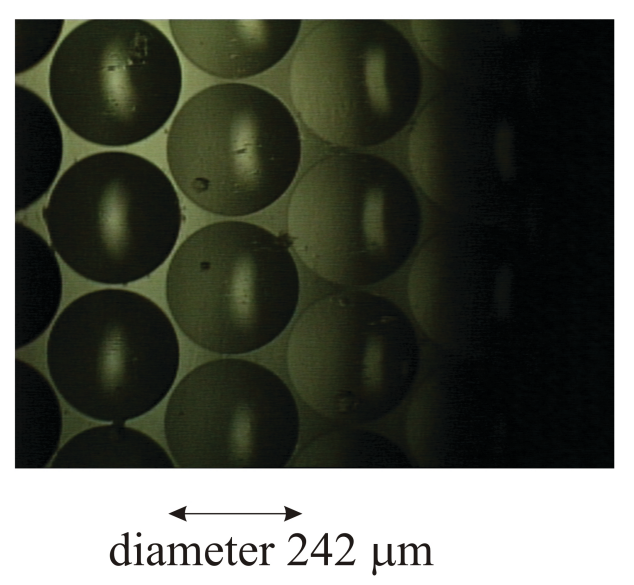

b)

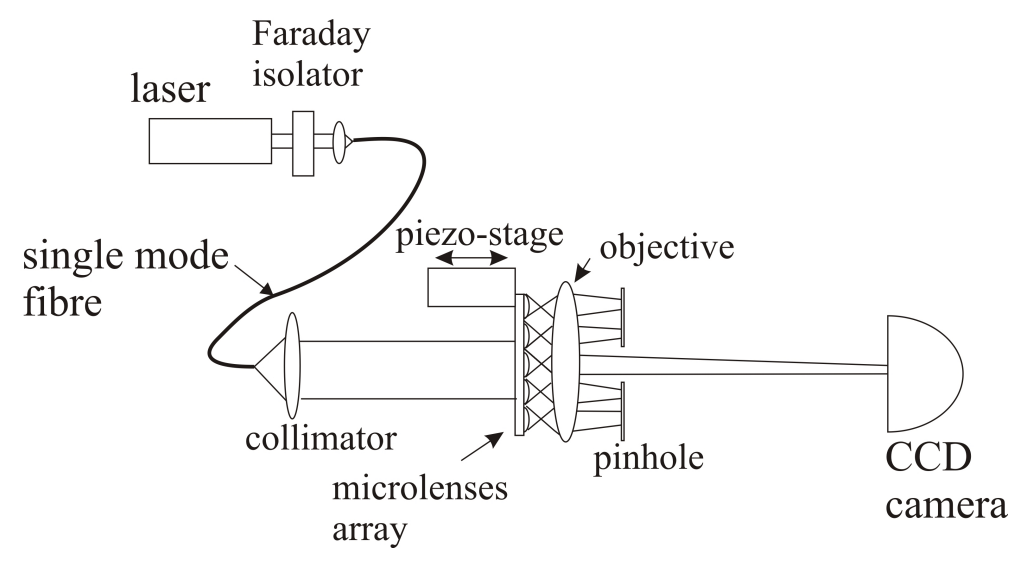

Figure 3. a) image of the top surface of the microlenses array. b) Experimental setup.

Another experimental issue regards the intensity and phase distribution of the field at the entrance of the microlenses. This field will, in general, not be perfectly uniform and this can result in additional aberrations found by the ENZ retrieval algorithm. Since we are only interested in the aberrations of the microlens being tested we should strive to keep the field at the entrance of the microlens as uniform as possible. To ensure uniform illumination, we apply a single mode fiber together with a low numerical aperture lens to further collimate the beam. In this way we can achieve a wavefront deviation of approximately $0.05 \lambda$ RMS in a beam of about $7 \mathrm{~mm}$ in diameter. Add up the fact that the individual microlenses are illuminated by only a minute fraction of the beam, we can thus consider the field at the entrance of the microlens to be uniform.

In figure $3 \mathrm{~b}$ we show the main optical path of the proposed setup. As seen in the figure, the collimated beam from the optical fiber illuminates the array of microlenses and the focal region is imaged onto a CCD camera with a high numerical aperture objective. If one takes a microscope objective of high numerical aperture. The distance between the microlenses and the objective is such to give a magnification factor at the CCD camera of about 100 times. Given this magnification, the imaged focused field at the camera occupies an area of about 100x100 pixels. As mentioned before, the knowledge of the exact magnification factor is important for the retrieval, and experimentally, the exact distances between the lenses and the camera cannot be measured with accuracy. In this case, the magnification should be measured independently by imaging at the CCD plane a calibrated object set in place of the microlens array. 

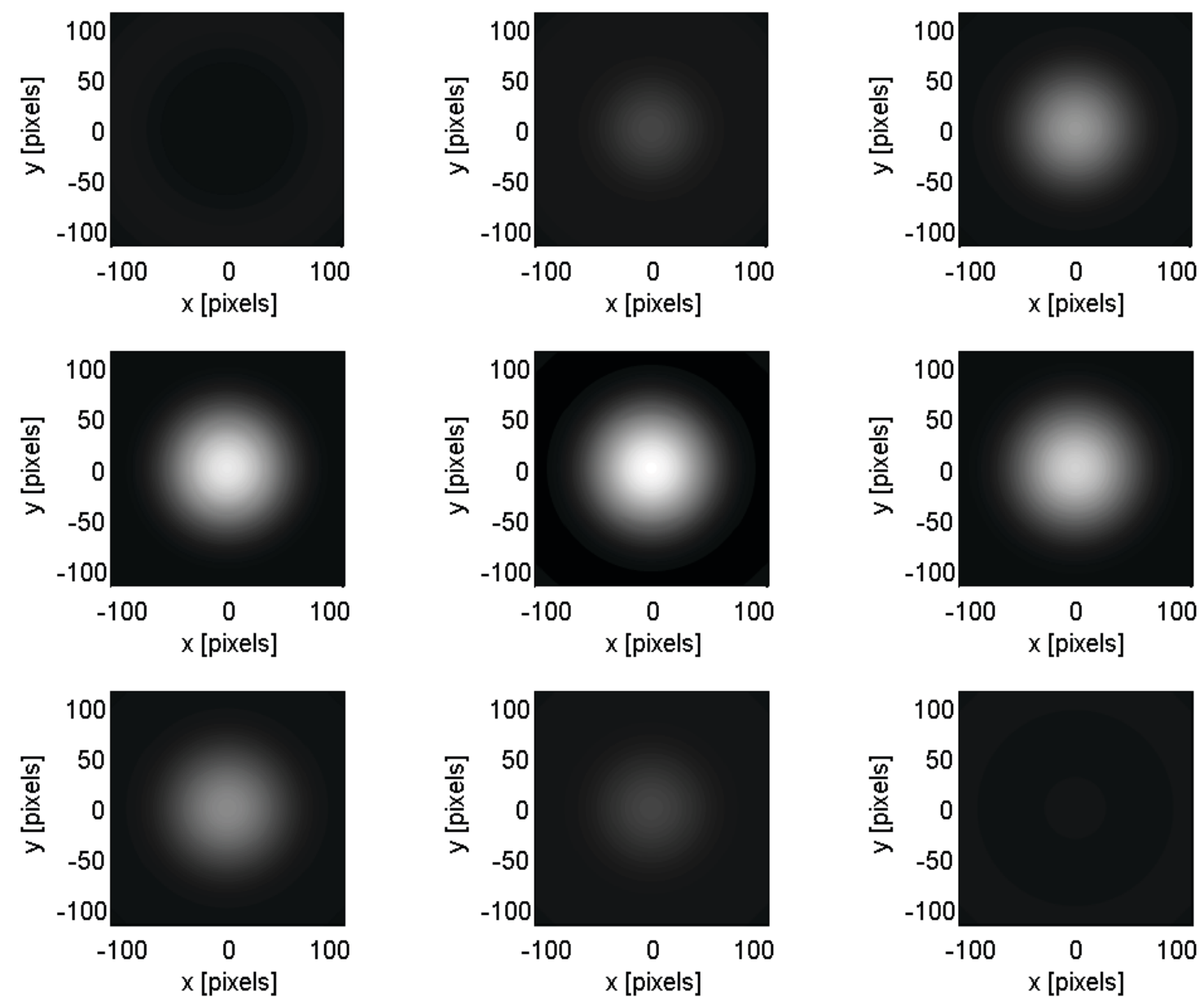

Figure 4. A simulated set of through-focus detector images pertaining to the measurement configuration proposed in this paper.

In order to record the through-focus images, we have chosen the approach where the distance between the imaging objective and the camera is kept fixed, while the array of microlenses is scanned in the axial distance. In this way, the magnification factor is always constant. Other approaches are also possible for example, displacing the axial position of the camera or that of the objective. Given that the distance between consecutive throughfocus planes should also be known with accuracy, the microlenses array should be mounted on a translator coupled to a piezo-electric stage with active feedback; this will ensure precision positioning of the axial distance.

One last issue on the design regards the response of the CCD camera. Most commercial cameras have adaptable gain that is automatically set in order to avoid saturation of the image. In our case, this cannot be used, since the relative intensity from the various through-focus images should be kept. Also, linearity of the response of the CCD camera over the entire dynamic range is important. If this is not the case, extra calibration should be made.

Considering the above proposed experimental configuration, we have simulated the through-focus intensity data that can be expected to be produced by this optical setup. A set of 9 simulated through-focus intensity images is included in Figure 4 showing that the number of relevant pixels for the analysis is more than sufficient. 


\section{CONCLUSIONS}

In conclusion, we have shown that the Extended Nijboer Zernike formalism can be applied to retrieve aberrations of micro-optics systems such as microlenses. The method is based on direct measurement of through focus images combined with computer retrieval procedure. In our proposed experiment, we have analyzed a scheme with an extra lens that magnifies the field in the focal region. This allows the use of a simple CCD camera to obtain the through focus images that relaxes the high lateral resolution required for direct imaging of the focused region and leads to great simplification of the experiment as compared to other schemes based on printing the focused field on resists or the use of near field apertures. The setup has been designed based on a real microlens array and its main issues have been presented and analyzed. The system requires the knowledge of the input field distribution at the microlens surface as well as the magnification factor of the image system used to magnify the focal region. Once these parameters have been calibrated, it becomes quite simple and quick to perform measurements of several lenses. Given its simplicity and precision, this method could be of interest for a wide range of applications in optics and micro-optics.

\section{ACKNOWLEDGMENTS}

This research is supported by NanoNed, a national nanotechnology program coordinated by the Dutch ministry of economic affairs. Furthermore, we acknowledge the Free University of Brussels for lending us the arrays of microlenses that have been used for this analysis, and also the European Network for Micro Optics (NEMO) that has been the starting point of this research.

\section{REFERENCES}

[1] Braat, J. J. M., van Haver, S., Janssen, A. J. E. M., and Dirksen, P., "Assessment of optical systems by means of point-spread functions," in [Progress in Optics], Wolf, E., ed., 51, ch. 6, 349-468, Elsevier B.V. (2008).

[2] Janssen, A. J. E. M., "Extended Nijboer-Zernike approach for the computation of optical point-spread functions," J. Opt. Soc. Am. A 19, 849-857 (2002).

[3] Braat, J., Dirksen, P., and Janssen, A. J. E. M., "Assessment of an extended Nijboer-Zernike approach for the computation of optical point-spread functions," J. Opt. Soc. Am. A 19, 858-870 (2002).

[4] Dirksen, P., Braat, J. J. M., Janssen, A. J. E. M., and Leeuwestein, A., "Aberration retrieval for high-NA optical systems using the extended Nijboer-Zernike theory," in [Proc. SPIE], 5754, 262-273 (May 2005).

[5] Dirksen, P., Braat, J. J. M., and Janssen, A. J. E. M., "Estimating resist parameters in optical lithography using the extended Nijboer-Zernike theory," J. Microlith., Microfabr., Microsyst. 5, 1-11 (Jan. 2006).

[6] van Haver, S., Braat, J. J. M., Dirksen, P., and Janssen, A. J. E. M., "High-NA aberration retrieval with the extended Nijboer-Zernike vector diffraction theory," J. Europ. Opt. Soc. Rap. Public. 1, 06004 (June 2006).

[7] Wolf, E. and Li, Y., "Conditions for the validity of the Debye integral representation of focused fields," Opt. Commun. 39(4), 205-210 (1981).

[8] Li, Y. and Wolf, E., "Three-dimensional intensity distribution near the focus in systems of different Fresnel numbers," J. Opt. Soc. Am. A 1(8), 801-808 (1984).

[9] Sheppard, C. J. R. and Török, P., "Focal shift and the axial optical coordinate for high-aperture systems of finite Fresnel number," J. Opt. Soc. Am. A 20(11), 2156-2162 (2003).

[10] Török, P., "An imaging theory for advanced, high-numerical-aperture optical microscopes." Thesis presented at the Hungarian Academy of Sciences (MTA Müszaki Fizikai Kutató Intézetében), 2004. 